Sharif University of Technology
Scientia Iranica
SCIENTIA
I RAN I CA

\title{
A new simplified formula in prediction of the resonance velocity for multiple masses traversing a thin beam
}

\author{
R. Afghani Khoraskani ${ }^{\mathrm{a}}$, M. Mofid ${ }^{\mathrm{b}}$, S. Eftekhar Azam ${ }^{\mathrm{c}}$ and \\ M. Ebrahimzadeh Hassanabadid,* \\ a. Faculty of Architecture and Urban Planning, Shahid Beheshti University, Tehran, Iran. \\ b. Department of Civil Engineering, Sharif Univeristy of Technology, Tehran, Iran. \\ c. Politecnico di Milano, Dipartimento di Ingegneria Civile e Ambientale, Piazza L. da Vinci 32, 20133 Milano, Italy. \\ d. Department of Structural Engineering, Road, Housing, and Urban Development Research Center, Tehran, Iran. \\ Received 29 September 2014; received in revised form 11 June 2015; accepted 25 July 2015
}

\author{
KEYWORDS \\ Euler-Bernoulli beam; \\ Dynamic response; \\ Series of traveling \\ masses; \\ Resonance; \\ Simplified formula.
}

\begin{abstract}
In this article, transverse vibration of an Euler-Bernoulli beam carrying a series of traveling masses is analyzed. A semi-analytical approach based on eigenfunction expansion method is employed to achieve the dynamic response of the beam. The inertia of the traveling masses changes the fundamental period of the base beam. Therefore, a comprehensive parametric survey is required to reveal the resonance velocity of the traversing inertial loads. In order to facilitate resonance detection for engineering practitioners, a new simplified formula is proposed to approximate the resonance velocity.

(C) 2016 Sharif University of Technology. All rights reserved.
\end{abstract}

\section{Introduction}

The investigations on moving load dynamic problems are said to be initiated early in the 19th century, ever since the first railway bridges were built [1]. Since then, numerous studies have discovered different aspects of this subject in structural dynamics [2-5]. The term moving load emphasizes the time-varying position of an applied load on a specific structural member/mechanical device [6-13].

The railways [14-16], bridges [17-25], ground [26], and pavements [27] could be mentioned as the wellknown cases of civil structures influenced by traveling loads. There exists a variety of sources producing moving loads on the structures such as the pedestri-

*. Corresponding author. Tel.: +98 2188255942 ; Fax: +982188254842

E-mail addresses: r.afghani@sbu.ac.ir (R. Afghani Khoraskani); mofid@sharif.edu (M. Mofid);

s.eftekharazam@stru.polimi.it (S. Eftekhar Azam); m.ebrahimzadeh@bhrc.ac.ir, and mohsen.eb.h@gmail.com (M. Ebrahimzadeh Hassanabadi) ans $[19,22,25]$, trains $[14,15]$, aircrafts $[3,4,11]$, or the moving vehicles [27].

The numerical explorations of bridges have been widely accomplished by the researchers assuming a beam element to establish the governing equations of motion $[1,7,8,12,28-35]$. Prediction of the dynamic performance of a bridge under a moving load would also be necessary in the health monitoring of the structure $[36,37]$ or the assessment of design parameters spectra $[8,11,20,24]$.

A moving load dynamic problem could be simulated either by the moving force [3,38-40] or the moving mass $[6,8,11,12,18,20,24,29,41,42]$ modeling assumptions. The moving force is a highly simplified model in which the vehicle/bridge interaction force is limited to the weight of structure. In contrast to the moving force approach, the moving mass framework yields valid results for a wider range of parameters leading to a more realistic assessment of the base structure dynamics; this is due to the consideration of inertial interaction of the traveling load and its substructure in the moving mass modeling approach. 
An issue of considerable interest in the bridge engineering is the detection of bridge resonant states caused by the multiple traversing loads [20,39-41]. The resonance leads to the ballast degradation, derailment, deterioration of passenger ride comfort, and increase in the maintenance costs [39]. Accordingly, it would be useful to predict the resonance velocity of a series of moving loads traversing the bridge; in this regard, the moving loads traversing the bridge at resonance velocity lead to resonant vibration of the structure.

Regarding the available literature on the vibration of bridges under moving vehicles, the prediction of moving masses resonance velocity is yet an issue open to discussion in the multiple moving mass problem. Dealing with the multiple moving forces, the system matrices are stationary and known. Hence, the exact resonance velocity could be analytically computed. However, in the case of the moving mass problem, matrices of the system continuously vary in time; therefore, it is not simple to analytically derive an exact formula for the resonance velocity as that of the multiple moving forces.

In this study, the problem of a series of identical and equally spaced moving masses travelling with the same speed along a beam-type structure is focused. This is frequently presumed as a model of a train passing over a bridge. Eigenfunction expansion method is exploited to capture the base-beam deflections. A new simplified formula is proposed to predict resonance velocity of the traversing masses. The formula is essentially based on the assumption that introducing the inertia of travelling masses into the mass matrix of the evolution equations will change the fundamental period of the structure; therefore, it results in a different resonance velocity than that obtained by neglecting the inertia of the masses (moving force approximation). The accuracy of the introduced relation is assessed through extensive numerical analyses, where the proposed formula shows a very close agreement with the true resonance.

\section{Problem formulation}

A uniform, continuous Euler-Bernoulli beam is considered as shown in Figure 1. It is assumed that both bending stiffness of the base beam $(E I)$ and the mass per unit length $(\rho A)$ are constant throughout the beam. Permanent contact condition between the

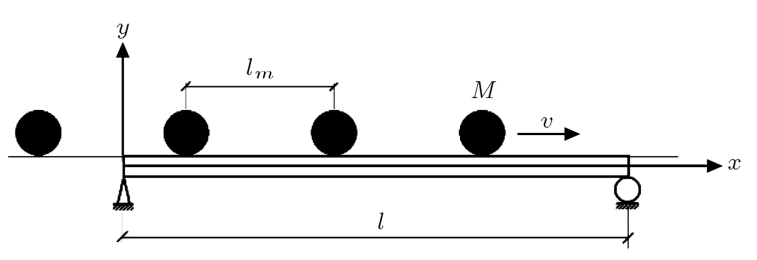

Figure 1. A beam excited by multiple moving masses. moving masses and the structure is assumed during the period of loads movement on the beam. Regarding the Euler-Bernoulli Beam theory, the constitutive equation of motion could be written as $[43,44]$ :

$$
\left\{\begin{array}{c}
E I \frac{\partial^{4}}{\partial x^{4}} W(x, t)+\rho A \frac{\partial^{2}}{\partial t^{2}} W(x, t) \\
\quad=-\sum_{k=1}^{N} \xi_{k} P_{k} \delta\left(x-X_{k}(t)\right), \\
P_{k}=M_{k}\left(g+\eta \frac{d^{2}}{d t^{2}} W\left(X_{k}(t), t\right)\right),
\end{array}\right.
$$

where $W$ is the beam deflection and its positive direction is assumed to obey the positive direction of the $y$ axis in Figure 1 (i.e. $+\uparrow$ ); the horizontal axis $x$ is assumed to correspond to the beam neutral axis; $\rho$ is mass per unit of volume; $A$ is the cross sectional area of the beam; and $t$ signifies time. Moreover, $M_{k}, g$, and $\delta()$ are mass of the $k$ th traveling object, gravitational acceleration, and Dirac delta, respectively. $X_{k}(t)$ and $P_{k}$ are the location and the contact force of the $k$ th traveling mass, and $N$ is the number of the moving loads. The function $\xi_{k}$ is defined as:

$$
\xi_{k}= \begin{cases}1, & 0 \leq X_{k}(t) \leq l \\ 0, & X_{k}(t)<0 \text { or } l<X_{k}(t)\end{cases}
$$

The parameter $\eta$ allows for convenient switching between moving mass and moving force frameworks. $\eta=$ 0 corresponds to the simplified moving force simulation and by assuming $\eta=1$, the full contribution of moving loads inertial interaction with the base beam could be considered in the mathematical model (moving mass). A semi analytical procedure based on eigenfunction expansion method could be adopted to treat Eq. (1). To this end, the beam equation of free vibration should be tackled to extract the eigenfunctions:

$$
E I \frac{\partial^{4} w_{j}(x)}{\partial x^{4}}=\rho A \omega_{j}^{2} w_{j}(x)
$$

where $w_{j}(x)$ denotes the $j$ th shape function, and $\omega_{j}$ is the $j$ th natural frequency. $W(x, t)$ in Eq. (1) could be replaced by a series expansion as:

$$
W(x, t)=\sum_{j=1}^{\infty} a_{j}(t) w_{j}(x) \approx \sum_{j=1}^{n} a_{j}(t) w_{j}(x) .
$$

Substituting Eq. (4) into Eq. (1) yields:

$$
\begin{aligned}
\sum_{j=1}^{n}\left\{\left[a_{j}(t) \omega_{j}^{2}+\frac{d^{2}}{d t^{2}} a_{j}(t)\right] \rho A w_{j}(x)\right\} \\
\quad=-\sum_{k=1}^{N} \xi_{k}\left(M_{k} g+\eta M_{k} \sum_{j=1}^{n} d^{2} / d t^{2}\left\{a_{j}(t) w_{j}\right.\right. \\
\left.\left.\quad\left(X_{k}(t)\right)\right\}\right) \delta\left(x-X_{k}(t)\right) .
\end{aligned}
$$

By regarding the orthonormal shape functions charac- 
teristic, i.e.:

$$
\int_{0}^{l} \rho A w_{i}(x) w_{j}(x) d x=\delta_{i j}= \begin{cases}0, & i \neq j \\ 1, & i=j,\end{cases}
$$

in which the shape functions are normalized by mass per unit length of the beam $\rho A$. Multiplying both sides of Eq. (5) by $w_{i}(x)$, and then integrating over the beam length yields:

$$
\begin{aligned}
\left(\omega_{i}^{2} a_{i}(t)\right. & \left.+\frac{d^{2}}{d t^{2}} a_{i}(t)\right)=-\sum_{k=1}^{N} \xi_{k}\left\{M_{k} g\right. \\
& \left.+\eta M_{k} \sum_{j=1}^{n} d^{2} / d t^{2}\left[a_{j}(t) w_{j}\left(X_{k}(t)\right)\right]\right\} \\
& w_{i}\left(X_{k}(t)\right) .
\end{aligned}
$$

The expanded form of $\frac{d^{2}}{d t^{2}}\left[a_{j}(t) w_{j}\left(X_{k}(t)\right)\right]$ is:

$$
\begin{aligned}
& \frac{d^{2}}{d t^{2}}\left(a_{j}(t) w_{j}\left(X_{k}\right)\right)=w_{j}\left(X_{k}\right) \frac{d^{2}}{d t^{2}} a_{j}(t) \\
& +2\left[\left(\frac{\partial w_{j}(x)}{\partial x}\right) \frac{d X_{k}}{d t}\right]_{x=X_{k}} \frac{d}{d t} a_{j}(t) \\
& +\left[\left(\frac{\partial^{2} w_{j}(x)}{\partial x^{2}}\right)\left(\frac{d X_{k}}{d t}\right)^{2}\right. \\
& \left.+\left(\frac{\partial w_{j}(x)}{\partial x}\right)\left(\frac{d^{2} X_{k}}{d t^{2}}\right)\right]_{x=X_{k}} a_{j}(t) .
\end{aligned}
$$

By assuming uniform moving masses $M_{k}=M$ with constant velocity $v$ as depicted in Figure 1, Eq. (8) could be simplified to:

$$
\begin{gathered}
w_{j}\left(X_{k}\right) \frac{d^{2}}{d t^{2}} a_{j}(t)+2 v\left(\frac{\partial}{\partial x} w_{j}(x)\right)_{x=X_{k}} \frac{d}{d t} a_{j}(t) \\
+v^{2}\left(\frac{\partial^{2}}{\partial x^{2}} w_{j}(x)\right)_{x=X_{k}} a_{j}(t),
\end{gathered}
$$

and the matrix from explanation of Eq. (7) could be written as:

$$
\mathbf{M}(t) \frac{d^{2}}{d t^{2}} \mathbf{a}(t)+\mathbf{C}(t) \frac{d}{d t} \mathbf{a}(t)+\mathbf{K}(t) \mathbf{a}(t)=\mathbf{F}(t),
$$

where:

$$
\begin{aligned}
& \mathbf{M}(t)=\left[\delta_{i j}\right]_{n \times n}+\eta \sum_{k=1}^{N} \mathbf{M}_{k}(t) \\
& \mathbf{M}_{k}(t)=\left[m_{i j}^{k}(t)\right]_{n \times n}=\xi_{k} M\left[w_{i}(x) w_{j}(x)\right]_{x=X_{k}} \\
& \mathbf{C}(t)=\eta \sum_{k=1}^{N} \mathbf{C}_{k}(t)
\end{aligned}
$$

$$
\begin{aligned}
\mathbf{C}_{k}(t) & =\left[c_{i j}^{k}(t)\right]_{n \times n} \\
& =\xi_{k} M\left[2 v w_{i}(x)\left(\frac{\partial}{\partial x} w_{j}(x)\right)\right]_{x=X_{k}}, \\
\mathbf{K}(t) & =\left[\omega_{i}^{2} \delta_{i j}\right]_{n \times n}+\eta \sum_{k=1}^{N} \mathbf{K}_{k}(t), \\
\mathbf{K}_{k}(t) & =\left[K_{i j}^{k}(t)\right]_{n \times n} \\
& =\xi M\left[v^{2} w_{i}(x) \frac{\partial^{2}}{\partial x^{2}} w_{j}(x)\right]_{x=X_{k}}, \\
\mathbf{F}(t) & =\sum_{k=1}^{N} \mathbf{F}_{k}(t), \\
\mathbf{F} & \\
\mathbf{a}(t) & =\left[f_{i}^{k}(t)\right]_{n \times 1}=-\xi_{k} M g\left[w_{i}\left(X_{k}(t)\right)\right], \\
\mathbf{a}(t)= & {\left[a_{i}(t)\right]_{n \times 1} . }
\end{aligned}
$$

The second order coupled ODEs, set in Eq. (9), can be reformulated to a reduced first order set of equations as follows:

$$
\begin{aligned}
& \frac{d}{d t} \mathbf{Q}(t)=\mathbf{A}(t) \mathbf{Q}(t)+\mathbf{G}(t), \\
& \mathbf{Q}\left(t_{0}\right)=\mathbf{Q}_{0}
\end{aligned}
$$

where:

$$
\begin{aligned}
& \mathbf{A}(t)=\left[\begin{array}{cc}
\mathbf{0}_{n \times n} & \mathbf{I}_{n \times n} \\
-\mathbf{M}^{-1} \mathbf{K} & -\mathbf{M}^{-1} \mathbf{C}
\end{array}\right]_{2 n \times 2 n}, \\
& \mathbf{Q}(t)=\left[\begin{array}{c}
\mathbf{a}(t) \\
\frac{d}{d t} \mathbf{a}(t)
\end{array}\right]_{2 n \times 1}, \\
& \mathbf{G}(t)=\left[\begin{array}{c}
\mathbf{0}_{n \times 1} \\
\mathbf{M}^{-1} \mathbf{F}
\end{array}\right]_{2 n \times 1}
\end{aligned}
$$

Eq. (19) can be solved by using matrix exponential [45]. In this study, the first three vibration modes are used in the analysis.

\section{The resonance velocity}

When a set of sequential moving masses travels along the beam length, at a certain velocity, the beam is expected to start to resonate. The most frequent resonance case is related to the coincidence of frequency content of excitation with the vibration frequency of the first deflection mode of the whole system. In the case that the inertial effects of the masses are negligible, the first natural frequency of the system can be reasonably assumed as that of the beam alone. 
However, the fundamental frequency of the base beam will change due to the inertial effects of the moving masses added to the system. To calculate the resonance velocity, due to the complications introduced by the inertia of the masses, these variations have usually been ignored; hence, the equations employed to predict the resonance velocity, previously, are frequently based on the moving force presumption.

In this study, the resonance velocity derived via moving force is modified so as to account for the inertial effects of the moving objects traveling over a beam-type structure. To accomplish this objective, the possible changes in the natural frequency of the system due to the inertial effects of the moving masses have been included in the analyses as well.

The resonance velocity of multiple moving forces traveling along the beam could be expressed as:

$$
v_{p}=\frac{l_{m} \omega_{1}}{2 j \pi},
$$

where $l_{m}$ is the spacing between the moving masses (Figure 1), and $\omega_{1}$ denotes the fundamental frequency of the base beam. Given the load spacing, $l_{m}$, the resonance speed for the series of moving forces becomes proportionate to $\frac{1}{j}$, where $j=1,2, \ldots$, which implies that resonance will take place at speeds starting from the primary resonance velocity $\frac{l_{m} \omega_{1}}{2 \pi}$ in descending values. To obtain the modified fundamental frequency of the beam carrying a series of moving inertial loads, it is assumed that the mass of the system is increased by an amount equal to $k . \beta . \rho . A . l$, while the stiffness of the system remains constant; where $\beta$ is the mass ratio of each moving object with respect to the beam mass, and $k$ is the maximum number of objects that can be simultaneously placed on the beam and will cause the maximum static deflection in the beam. In other words, a beam with the same flexural stiffness, EI, but with an increased uniformly distributed mass should be considered to approximate the fundamental frequency of the system. Consequently, assuming a beam with mass per unit volume of:

$$
\rho^{\prime}=(k \cdot \beta+1) \cdot \rho,
$$

and regarding the fact that the first natural frequency of a thin beam is proportional to $\sqrt{\frac{E I}{\rho A}}[46]$, the fundamental frequency of the assumed beam with increased mass density, $\rho^{\prime}$, could be attained by a modification factor:

$$
\left\{\begin{array}{l}
\omega_{1}^{\prime}=\alpha \omega_{1}, \\
\alpha=\sqrt{\frac{1}{1+k \beta}} .
\end{array}\right.
$$

By applying this modification factor to Eq. (23), the resonance velocity for multiple moving masses could be approximately simplified as follows:

$$
v_{r e s}=\sqrt{\frac{1}{1+k \beta}} v_{p} .
$$

Considering a simply supported single span beam $\omega_{i}=$ $\left(\frac{i \pi}{l}\right)^{2} \sqrt{\frac{E I}{\rho A}}$, for the largest resonance velocity (primary resonance velocity), Eq. (26) reads:

$$
v_{\text {res }}=\sqrt{\frac{1}{1+k \beta}}\left(\sqrt{\frac{E I}{\rho A}} \frac{\pi l_{m}}{2 l^{2}}\right) .
$$

\section{Numerical examples}

A single span simply-supported Euler-Bernoulli beam is considered for the numerical examples. The boundary conditions of the beam are $W(0, t)=\frac{\partial^{2}}{\partial x^{2}} W(0, t)=$ 0 and $W(l, t)=\frac{\partial^{2}}{\partial x^{2}} W(l, t)=0$. Therefore, normalized eigenfunctions of the beam, considering Eq. (6), could be given by $w_{i}(x)=\sqrt{\frac{2}{\rho A l}} \sin \left(\frac{i \pi x}{l}\right)$. The coupled dynamic system of beam and moving masses are supposed to be at rest at the beginning moment of analysis $t=0$; therefore, zero initial condition is considered for Eq. (1) i.e. $W(x, 0)=0$ and $\left.\frac{\partial}{\partial t} W(x, t)\right|_{t=0}=0$. A customary standard normalization scheme $[4,5,8]$ is adopted in the representation of results by which the outputs are described with non-dimensional relative parameters. The deflection of the beam at its midspan is selected as representative for the quantities of the beam response; moreover, throughout the article, the maximum static deflection caused by a single load in the set of the relevant travelling masses, $W_{\text {stat }}=\frac{-M g l^{3}}{48 E I}$, is used to present normalized deflection, $W_{N}=W(0.5 l, t) / W_{\text {stat }}$. Such normalization provides a better understanding of the dynamic amplification factor, $\mathrm{DAF}=\max \left(W_{N}\right)$, which is the maximum value of beam normalized deflection, $W_{N}$, during the forced vibration of the beam.

To numerically determine the critical velocity, a range of values of $v_{p}$ has been considered, and the dynamic amplification factors are obtained for each velocity at which the series of masses pass over the beam. The spectral analyses are presented in Figure 2, wherein the abscissa denotes normalized velocity $v / v_{p}$ and the vertical axis represents the associated Dynamic Amplification Factor (DAF). In Figure 2, the results of the aforementioned analyses are confronted for the two cases of moving forces and moving masses. The resonance velocity in this manner is the velocity at which the peaks of DAF take place. For the series of moving forces, the maximum of DAF peak appears at the primary resonance velocity, i.e. $v=v_{p}$ which elucidates Eq. (23). As mentioned before, resonance will take place at speeds starting from a primary 


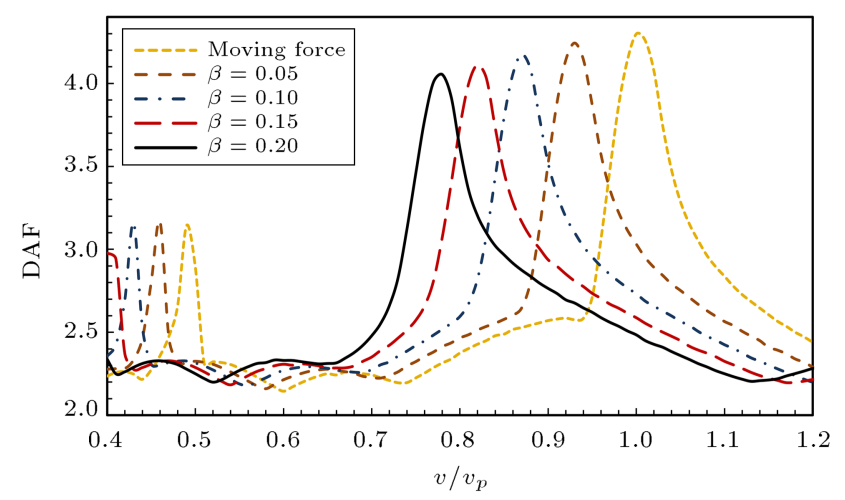

Figure 2. Dynamic amplification factor at the midspan of the beam at various travelling velocities. Moving force versus moving mass, $N=15, l_{m}=0.3 l$.

resonance velocity, then, repeating in descending values proportional to $\frac{1}{j}$ of the primary resonance, where $j=1,2, \ldots$ The spike which appears at $v=0.5 v_{p}$ is associated with the secondary resonance velocity. A shifting trend of the spectral curves towards lower resonance frequency components could be observed as the mass of the moving objects increases. This would strictly justify the fact that the loads inertia can notably dominate either the primary or the secondary resonance velocities.

In case the ratio of the mass is not small as compared to the beam mass, the resonance velocity obtained for moving forces will be of poor accuracy for the multiple moving mass problem; for instance, in case the ratio of the mass of travelling objects and the beam mass $\beta=\frac{M}{\rho A l}$ are equal to 0.15 , the resonance velocity turns out to be $0.83 l v_{p}$ or, equivalently, $\alpha=$ 0.831. Therefore, as the mass ratio of the travelling masses increases, the modification in the moving forces resonance velocity becomes increasingly significant.

Without any loss of generality, let place our focus on the resonance speed at which the maximum peak of

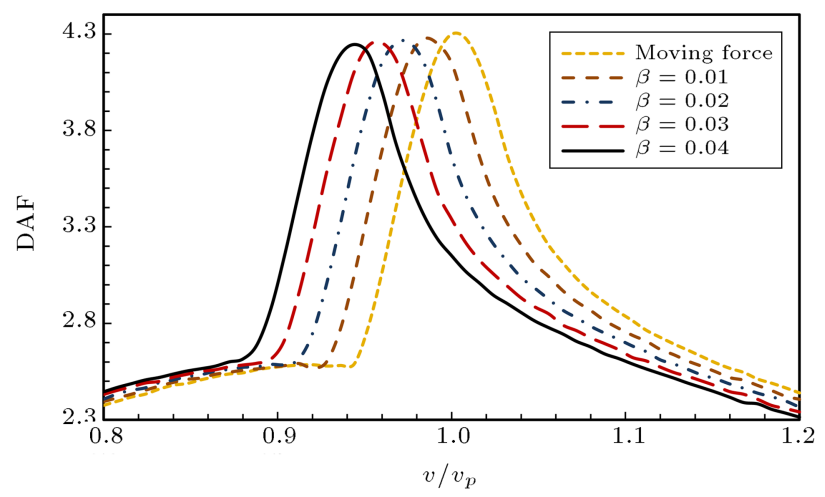

Figure 3. Dynamic amplification factor at the midspan of the beam at various travelling velocities. Moving force versus moving mass, $N=15, l_{m}=0.3 l$.

DAF, or, in other words, the so-called herein primary resonance velocity is produced. In Figure 3 , the later DAF spectral analysis is inspected for a series of moving masses, but instead, with small mass ratio with concentration on the primary resonance. It could be observed that even for relatively light moving masses, namely $\beta=0.02$, the discrepancy of the moving force and moving mass at resonant states is not negligible.

To allow an assessment of the proposed formula for the resonance velocity with the numerical analyses, in a series of examples, the modification factors resulted from the numerical simulations are compared with the proposed simplified formula in Eq. (26), and the results of the comparison are presented in Table 1 . It could be observed that the resonance velocities obtained from Eq. (26) are in reasonable agreement with the resonance velocity attained by the spectral peaks in numerical analyses.

When there are 15 and 25 loads spaced by $l_{m}=$ $0.3 l$, for the mass ratio $\beta=0.2$, the difference between the two resonance velocities is $1.3 \%$. It could be perceived that the change in the number of moving

Table 1. Modification factors obtained by Eq. (26) and the spectra.

\begin{tabular}{|c|c|c|c|c|c|}
\hline $\begin{array}{l}\text { Number of } \\
\text { moving } \\
\text { masses }(\mathrm{N})\end{array}$ & $\begin{array}{c}\text { load } \\
\text { spacing } \\
\left(l_{m}\right)\end{array}$ & $k$ & $\boldsymbol{\beta}$ & $\begin{array}{l}\alpha \text { resulted } \\
\text { from } \\
\text { Eq. }(25) \\
\end{array}$ & $\begin{array}{c}\alpha \text { resulted } \\
\text { from } \\
\text { spectra } \\
\end{array}$ \\
\hline \multirow{3}{*}{15} & \multirow{3}{*}{$l_{m}=0.3 l$} & \multirow{3}{*}{3} & 0.10 & 0.877 & 0.884 \\
\hline & & & 0.15 & 0.830 & 0.831 \\
\hline & & & 0.20 & 0.791 & 0.785 \\
\hline \multirow{3}{*}{15} & \multirow{3}{*}{$l_{m}=0.6 l$} & \multirow{3}{*}{2} & 0.10 & 0.913 & 0.921 \\
\hline & & & 0.15 & 0.877 & 0.889 \\
\hline & & & 0.20 & 0.845 & 0.851 \\
\hline \multirow{3}{*}{25} & \multirow{3}{*}{$l_{m}=0.3 l$} & \multirow{3}{*}{3} & 0.10 & 0.877 & 0.874 \\
\hline & & & 0.15 & 0.830 & 0.824 \\
\hline & & & 0.20 & 0.791 & 0.775 \\
\hline
\end{tabular}




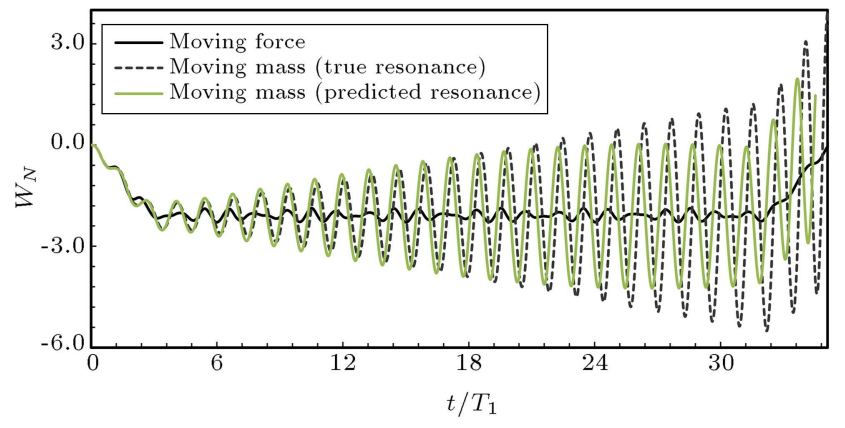

Figure 4. Time histories of the dynamic amplification factor at the midspan of a beam subjected to $N=25$ travelling masses at the true resonance velocity, the predicted resonance velocity, and the resonance velocity obtained from the moving force assumptions; $l_{m}=0.3 l$, $\beta=0.2$.

masses from $N=15$ to $N=25$ is influential in the computation of the true resonance velocities through the numerical method; the reason for this can be understood from Figure 4, wherein the time history of the structural dynamic response for a moving mass traveling at its true resonance velocity is depicted. At $t=0$, the first mass is at the left end of the beam, i.e. $x=0$. The beam experiences steady state loading scheme due to the stream of dynamic moving objects acting on the beam as long as the third mass enters the beam. This steady state dynamic loading continues until the $N$ th load reaches the left end of the beam. In this regard, by increasing the total number of moving masses $N$, the steady state part of the response would dominate the numerically computed true resonance velocity for which the predicted true resonance would tend towards its asymptotic state for $N=\infty$. The simplified formula is not sensitive to the total number of masses. It predicts the same value independent of this number, as does the original formula for resonance velocity of the set of moving forces.

In Figure 4, it can also be observed that although the behavior of the system at the resonance velocity predicted by the Eq. (26) does not exactly match the behavior of the system at the resonance velocity, it is far closer than the behavior of the system at the resonance velocity predicted without including the inertia of the masses in analysis $\left(v_{p}\right)$.

In Figure 5, the same time histories of Figure 4 are re-plotted assuming different weights of the moving masses, i.e. $\beta=0.1$. It could be seen that the values of the time histories and the maximum response for the case of true resonance velocity and the predicted resonance velocity via Eq. (26) are in close agreement, while the moving force is noticeably error-prone $\left(T_{1}\right.$ denotes the fundamental period of the beam).

According to Table 1, the precision of the simplified formula could be affected by the maximum number of masses which can be simultaneously placed on the

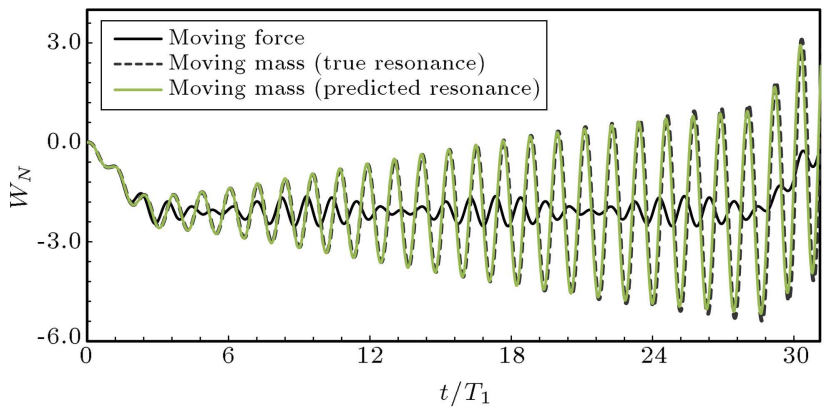

Figure 5. Time histories of the beam dynamic response subject to $N=25$ travelling masses at the true resonance velocity, the predicted resonance velocity, and the resonance velocity obtained from the moving force assumptions; $l_{m}=0.3 l, \beta=0.1$.

beam and the mass of the moving objects, i.e. $k$, which itself is dependent on spacing of the loads. With large spacing of the loads, $k$ reduces and it is important to mention that the stream of moving masses should travel at higher velocities to reach the primary resonance velocity. Therefore, the impact of the convective terms of the moving masses transverse acceleration (as mentioned in Eq. (8)) significantly increases. On the other hand, larger masses could amplify these effects. Accordingly, the simplified formula would feature small discrepancies with true resonance velocity.

In some analytical investigations, it has been emphasized that the moving mass and moving oscillator could be in close agreement for some real cases of vehicle bridge coupled systems [9]. A comparison of the present method provided by moving mass model with the corresponding moving oscillator problem (Figure 6) is depicted in Figure 7 . The method of investigation employed is that proposed by Yang et al. [47]. It can be concluded that the present technique is of good engineering level of agreement for suspension systems having $\Omega=\frac{\sqrt{K / M}}{\omega_{1}} \geq 2.0$.

\section{Conclusion}

In this paper, the constitutive equation of an EulerBernoulli beam acted upon by multiple moving masses is studied. A critical velocity is proposed in terms of the modified fundamental period of the structure, span length, and spacing of the moving masses. The

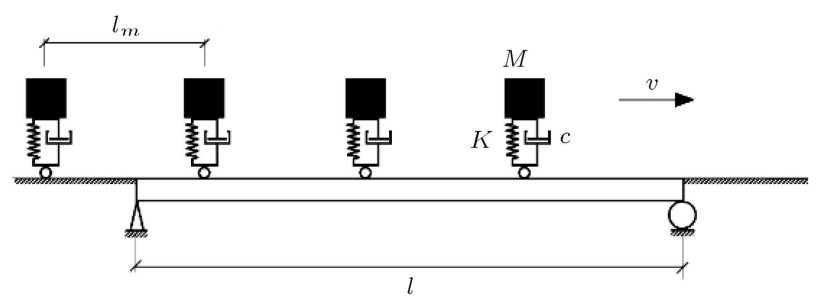

Figure 6. A beam traversed by sequential traveling oscillators. 


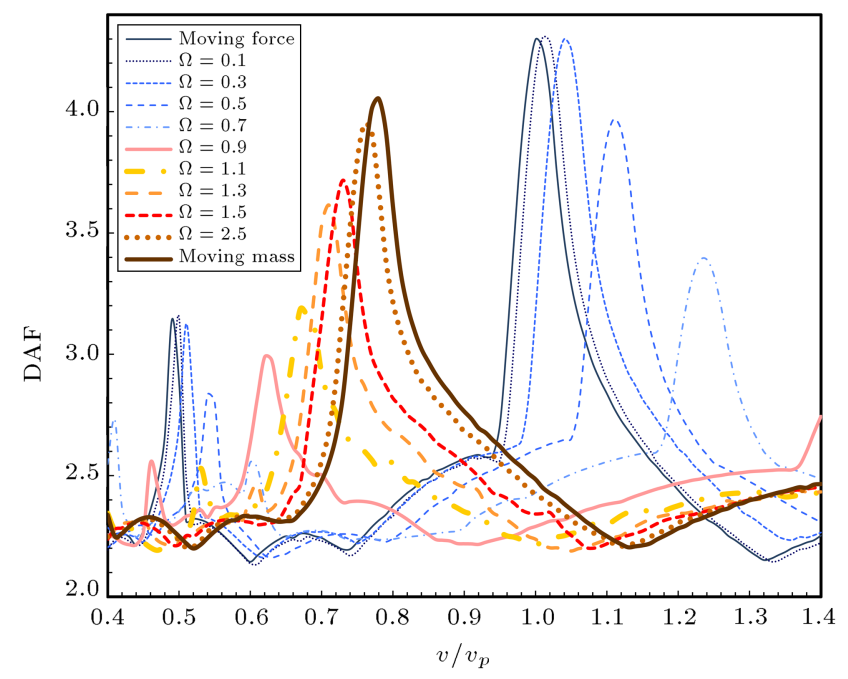

Figure 7. Dynamic amplification factor of the beam at various travelling velocities. Moving mass versus moving oscillator, $N=15, c=0, l_{m}=0.3 l, \beta=0.20$.

proposed resonance velocity is based on the resonance velocity of a beam subjected to a series of moving forces, which is rectified by a relevant modification factor. The results for approximate modification factor appear to be in reasonable agreement with the true ones obtained from numerical assessments. Moreover, regarding the vehicle suspension with $\Omega \geq 2.0$, a close agreement could be observed with the present method.

\section{References}

1. Olsson, M. "On the fundamental moving load problem", Journal of Sound and Vibration, 145(2), pp. 299-307 (1990).

2. Timoshenko, S.P., History of Strength of Materials: With a Brief Account of the History of Theory of Elasticity and Theory of Structures, McGraw-Hill, New York (1953).

3. Frýba, L., Vibration of Solids and Structures under Moving Loads, Thomas Telford, London (1999).

4. Ouyang, H. "Moving-load dynamic problems: A tutorial (with a brief overview)", Mechanical Systems and Signal Processing, 25(6), pp. 2039-2060 (2011).

5. Bajer, C.I. and Dyniewicz, B., Numerical Analysis of Vibrations of Structures Under Moving Inertial Load, Springer-Verlag, Berlin (2012).

6. Gbadeyan, J.A. and Dada, M.S. "Dynamic response of a Mindlin elastic rectangular plate under a distributed moving mass", International Journal of Mechanical Sciences, 48(3), pp. 323-340 (2006).

7. Omenzetter, P. "Interaction of a non-self-adjoint onedimensional continuum and moving multi-degree-offreedom oscillator", Journal of Sound and Vibration, 331(4), pp. 833-848 (2012).

8. Eftekhar Azam, S., Mofid, M. and Afghani Khoraskani,
R. "Dynamic response of Timoshenko beam under moving mass", Scientia Iranica, Transactions A: Civil Engineering, 20(1), pp. 50-56 (2013).

9. Ebrahimzadeh Hassanabadi, M., Vaseghi Amiri, J. and Davoodi, M.R. "On the vibration of a thin rectangular plate carrying a moving oscillator", Scientia Iranica, Transactions A: Civil Engineering, 21(2), pp. 284-294 (2014).

10. Hassanabadi, M.E., Attari, N.K.A., Nikkhoo, A. and Baranadan, M. "An optimum modal superposition approach in the computation of moving mass induced vibrations of a distributed parameter system", Proceedings of the Institution of Mechanical Engineers, Part C: Journal of Mechanical Engineering Science, 229(6), pp. 1015-1028 (2015).

11. Amiri, J.V., Nikkhoo, A., Davoodi, M.R. and Hassanabadi, M.E. "Vibration analysis of a Mindlin elastic plate under a moving mass excitation by eigenfunction expansion method", Thin-Walled Structures, 62, pp. 53-64 (2013).

12. Ebrahimzadeh Hassanabadi, M., Nikkhoo, A., Vaseghi Amiri, J. and Mehri, B. "A new orthonormal polynomial series expansion method in vibration analysis of thin beams with non-uniform thickness", Applied Mathematical Modelling, 37(18-19), pp. 8543-8556 (2013).

13. Yas, M.H. and Heshmati, M. "Dynamic analysis of functionally graded nanocomposite beams reinforced by randomly oriented carbon nanotube under the action of moving load", Applied Mathematical Modelling, 36, pp. 1371-1394 (2012).

14. Ang, K.K. and Dai, J. "Response analysis of highspeed rail system accounting for abrupt change of foundation stiffness", Journal of Sound and Vibration, 332, pp. 2954-2970 (2013).

15. Hung, H.H., Chen, G.H. and Yang, Y.B. "Effect of railway roughness on soil vibrations due to moving trains by $2.5 \mathrm{D}$ finite/infinite element approach", Engineering Structures, 57, pp. 254-266 (2013).

16. Dimitrovová, Z. and Rodrigues, A.F.S. "Critical velocity of a uniformly moving load", Advances in Engineering Software, 50, pp. 44-56 (2012).

17. Calçada, R., Cunha, A. and Delgado, R. "Analysis of traffic-induced vibrations in a cable-stayed bridge. Part I: Experimental assessment", Journal of Bridge Engineering, 10(4), pp. 370-385 (2005).

18. Eftekhari, S.A., Jafari, A.A. "Vibration of an initially stressed rectangular plate due to an accelerated traveling mass", Scientia Iranica, Transactions A: Civil Engineering, 19(5), pp. 1195-1213 (2012).

19. Piccardo, G. and Tubino, F. "Equivalent spectral model and maximum dynamic response for the serviceability analysis of footbridges", Engineering Structures, 40, pp. 445-456 (2012).

20. Nikkhoo, A., Ebrahimzadeh Hassanabadi, M., Eftekhar Azam, S. and Vaseghi Amiri, J. "Vibration 
of a thin rectangular plate subjected to series of moving inertial loads", Mechanics Research Communications, 55, pp. 105-113 (2014).

21. Kim, J. and Lynch, J.P. "Experimental analysis of vehicle-bridge interaction using a wireless monitoring system and a two-stage system identification technique", Mechanical Systems and Signal Processing, 28, pp. 3-19 (2012).

22. Ingólfsson, E.T., Georgakis, C.T. and Jönsson, J. "Pedestrian-induced lateral vibrations of footbridges: A literature review", Engineering Structures, 45, pp. 21-52 (2012).

23. Yi, T.H., Li, H.N. and Gu, M. "Experimental assessment of high-rate GPS receivers for deformation monitoring of bridge", Measurement, 46, pp. 420-432 (2013).

24. Nikkhoo, A., Farazandeh, A., Ebrahimzadeh Hassanabadi, M., Mariani, S. "Simplified modeling of beam vibrations induced by a moving mass by regression analysis", Acta Mechanica, 226(7), pp. 2147-2157 (2015).

25. Carroll, S.P., Owen, J.S. and Hussein, M.F.M. "A coupled biomechanical/discrete element crowd model of crowd-bridge dynamic interaction and application to the Clifton suspension bridge", Engineering Structures, 49, pp. 58-75 (2013).

26. Lefeuve-Mesgouez, G. and Mesgouez, A. "Threedimensional dynamic response of a porous multilayered ground under moving loads of various distributions", Advances in Engineering Software, 46, pp. 75-84 (2012).

27. Beskou, N.D. and Theodorakopoulos, D.D. "Dynamic effects of moving loads on road pavements: A review", Soil Dynamics and Earthquake Engineering, 31, pp. 547-567 (2011).

28. Lee, U. "Revisiting the moving mass problem: Onset of separation between the mass and beam", Journal of Vibration and Acoustics, 118(3), pp. 516-521 (1996).

29. Bulut, H. and Kelesoglu, O. "Comparing numerical methods for response of beams with moving mass", Advances in Engineering Software, 41, pp. 976-980 (2010).

30. Roshandel, D., Mofid, M. Ghannadiasl, A. "Modal analysis of the dynamic response of Timoshenko beam under moving mass", Scientia Iranica, Transactions A: Civil Engineering, 22(2), pp. 331-344 (2015).

31. Azizi, N., Saadatpour, M.M. and Mahzoon, M. "Using spectral element method for analyzing continuous beams and bridges subjected to a moving load", Applied Mathematical Modelling, 36, pp. 3580-3592 (2012).

32. Bowe, C.J. and Mullarkey, T.P. "Unsprung wheelbeam interactions using modal and finite element models", Advances in Engineering Software, 39, pp. 911-922 (2008).
33. Lotfollahi-Yaghin, M.A., Kafshgarkolaei, H.J., Allahyari, H. and Ghazvini, T. "On the absolute maximum dynamic response of a beam subjected to a moving mass", Structural Engineering and Mechanics, 54(1), pp. 55-67 (2015).

34. Roshandel, D., Mofid, M. and Ghannadiasl, A. "Dynamic response of a non-uniform Timoshenko beam, subjected to moving mass", Proceedings of the Institution of Mechanical Engineers, Part C: Journal of Mechanical Engineering Science, 229(14), pp. 24992513 (2015).

35. Nikkhoo, A., Farazandeh, A. and Hassanabadi, M.E. "On the computation of moving mass/beam interaction utilizing a semi-analytical method", Journal of the Brazilian Society of Mechanical Sciences and Engineering (2014). DOI : 10.1007/s40430 - $014-$ $0277-1$.

36. Khorram, A., Bakhtiari-Nejad, F. and Rezaeian, M. "Comparison studies between two wavelet based crack detection methods of a beam subjected to a moving load", International Journal of Engineering Science, 51, pp. 204-215 (2012).

37. Zhan, J.W., Xia, H., Chen, S.Y. and De Roeck, G. "Structural damage identification for railway bridges based on train-induced bridge responses and sensitivity analysis", Journal of Sound and Vibration, 330, pp. 757-770 (2011).

38. Uzal, E. and Sakman, L.E. "Dynamic response of a circular plate to a moving load", Acta Mechanica, 210(3-4), pp. 351-359 (2010).

39. Martínez-Rodrigo, M.D., Lavado, J. and Museros, P. "Dynamic performance of existing high-speed railway bridges under resonant conditions retrofitted with fluid viscous dampers", Engineering Structures, 32, pp. 808828 (2010).

40. Martínez-Rodrigo, M.D., Lavado, J. and Museros, P. "Transverse vibrations in existing railway bridges under resonant conditions: Single-track versus doubletrack configurations", Engineering Structures, 32, pp. 1861-1875 (2010).

41. Lu, Y., Mao, L. and Woodward, P. "Frequency characteristics of railway bridge response to moving trains with consideration of train mass", Engineering Structures, 42, pp. 9-22 (2012).

42. Esen, İ. "A new finite element for transverse vibration of rectangular thin plates under a moving mass", Finite Elements in Analysis and Design, 66, pp. 26-35 (2013).

43. Yang, Y.B., Yau, J.D. and Wu, Y.S., VehicleBridge Interaction Dynamics: With Applications to High-Speed Railways., World Scientific (2004). ISBN 9789812388476 .

44. Dimitrovová, Z. "On the moving mass versus moving load problem", IX International Conference on Structural Dynamics (EURODYN2014), 30, June - 2 July, Porto, Portugal (2014). 
45. Nikkhoo, A. and Rofooei, F.R. "Parametric study of the dynamic response of thin rectangular plates traversed by a moving mass", Acta Mechanica, 223(1), pp. 15-27 (2012).

46. Leissa, A.W. and Qatu, M.S., Vibration of Continuous Systems, McGraw Hill, New York (2011).

47. Yang, B., Tan, C.A. and Bergman, L.A. "Direct numerical procedure for solution of moving oscillator problems", Journal of Engineering Mechanics, 126(5), pp. $462-469$ (2000).

\section{Biographies}

Roham Afghani Khoraskani received his $\mathrm{PhD}$ degree from the Department of Building and Environmental Science and Technology (BEST) at Politecnico di Milano, Italy. He received his BS degree from Isfahan University of Technology (IUT) and his MS Degree from Sharif University of Technology (SUT), Iran. Currently, he is an Assistant Professor at Faculty of Architecture and Urban Planning, Shahid Beheshti University, Tehran, Iran.

Massood Mofid is Professor of Civil and Structural engineering. His research interests are in the area of structural analysis and structural dynamics.

Saeed Eftekhar Azam received his $\mathrm{PhD}$ degree from the Department of Structural engineering at Politecnico di Milano, Italy. He received his BS degree from Tehran University and his MS degree from Sharif University of Technology (SUT), Iran. His research interests include dynamics of structures, structural system identification, and structural health monitoring.

Mohsen Ebrahimzadeh Hassanabadi received his MS degree from the Department of Structural Engineering at Babol University of Technology, Iran, and his BS from University of Tehran, Iran. Currently, he is a researcher at BHRC. 